\title{
Zeno effect in spontaneous decay induced by coupling to an unstable level
}

\author{
Alfredo Luis* \\ Departamento de Óptica, Facultad de Ciencias Físicas, Universidad Complutense, 28040 Madrid, Spain
}

(Received 12 December 2000; published 13 August 2001)

\begin{abstract}
A metastable atomic level can be rendered unstable in a controllable way by coupling it to a decaying state. In this work we carry out a full dynamical analysis of the Zeno effect in this kind of unstable systems, comparing it to the inhibition of purely coherent Rabi oscillations. Simple and experimentally feasible measuring strategies involving three atomic levels are considered. It is shown that this induced decay is actually an example of a partial Zeno effect so that the observed evolution results from the competition of two Zeno effects. We also show that a three-level scheme can display both coherent, incoherent, and anti-Zeno effects.
\end{abstract}

DOI: 10.1103/PhysRevA.64.032104

\section{INTRODUCTION}

The Zeno effect was originally introduced as the inhibition of the irreversible decay of an unstable system caused by frequent enough observation [1]. Since then, this quantum phenomenon has found modifications and extensions such as the anti-Zeno [2,3] and the inverse-Zeno [4] effects, for example.

Despite its original definition, most of the theoretical and experimental efforts have focused on the inhibition of coherent reversible processes such as the Rabi oscillation in twolevel atoms as the most remarkable example (we will refer to this as the coherent Zeno effect) [5-7]. This is natural since closed systems are simpler than open ones. As a matter of fact, the practical implementation of this effect in decaying systems (we will refer to it as the incoherent Zeno effect) encounters difficulties and is very hard to observe (nevertheless, an experimental observation has been already reported in Ref. [8]). The difficulties arise because in order to stop the evolution the measurement must be performed during the initial stages of the decay, when the population still decreases quadratically with time. This is the period suitable for the Zeno effect. After this short-time interval the decay enters the exponential regime where no Zeno effect occurs. For most practical situations the initial stage of the evolution sensitive to measurement occurs so fast that extremely precise measurements would be required to stop the dynamics.

In order to overcome this difficulty it has been proposed to engineer the decay of an otherwise stable atomic level [9]. This consists of coherently coupling the stable state to a decaying one. Thus, the previously stable state becomes unstable. The key point is that the decaying parameters can be tailored. In particular this allows us to prolong the initial stage of the decay where the population decreases quadratically with time. This idea can be regarded as an example of reservoir engineering [10] and from now on we will refer to it as engineered decay.

It might be questioned whether engineered decay actually fits the original definition of the Zeno effect. This is because the initial stage of the evolution is a coherent Rabi oscillation. Therefore, it might be argued that these arrangements

*Electronic address: alluis@eucmax.sim.ucm.es
PACS number(s): 03.65.Xp, 42.50.-p, 32.80.- $-\mathrm{t}$

are no more than particular examples of the coherent Zeno effect.

The purpose of this work is to study these points in some detail looking for similarities and differences between the Zeno effect in engineered decay and in purely coherent evolutions. For the sake of clarity we focus on a feasible arrangement with three atomic levels in the $\mathrm{V}$ configuration which has been already used to demonstrate the coherent Zeno effect [5]. This choice has several advantages. It has been throughly studied, so its analysis can benefit from previous works [11-23] throwing new findings into relief. No less important is that the possibility of an experimental implementation is granted $[5,14]$. We will also show that for some parameter regime this same scheme provides an accessible simple example of an anti-Zeno effect, i.e., the speeding up of evolution caused by observation [2,3]. In order to accomplish these objectives we find and solve the evolution equations for the density matrix of the system, including the engineered decay and the continuous monitoring of the population of the initial state. This dynamical description is expressed by means of Bloch equations.

It must be noted that in this context the density matrix represents ensembles of independent systems that are observed simultaneously (or the average of repeated observations of a single system). This was actually the practical situation in the experiments reported in Refs. [5-7]. A relevant feature of this ensemble approach is that the evolution can be understood in common dynamical terms without ever resorting to controversial items such as the state reduction $[12,13,15,18,24]$. This is because the equations of motion for the system are obtained after discarding all the information provided by the apparatus. The evolution is then independent of the measurement results (nonselective evolution).

This approach has been criticized by arguing that the apparent divorce from measurement and state reduction veils the quantum microscopic origin of the effect [7,16,19-21]. On the other hand, the evolution of individual systems under the effect of measurement has been examined, both theoretically $[13,16,20,22]$ and experimentally [21]. Such an approach may be regarded as being closer to the original idea of the Zeno effect since the state of the system depends on the random outcomes of the measurement (selective evolution). It has been shown that for suitable parameter regimes the statistics of the results (usually in the form of random jumps [14]) embody the signature of state reductions caused 


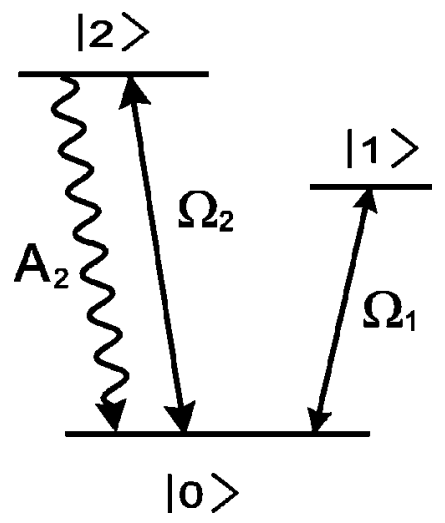

FIG. 1. Three-level scheme in the V configuration.

by measurement. It is worth pointing out that during the whole process the system is always in a pure state that depends on the history of the observed outcomes (quantum trajectory).

These two approaches are not independent. The average of many quantum trajectories tends to the solution of the Bloch equations. Also, the density matrix and the quantum trajectories would coincide exactly (without involving any averaging) in the ideal limit of a perfect Zeno effect. In such a case the system is always in the initial state with full certainty. Thus, the density matrix can be regarded as a coarsegrained following of the process avoiding the randomness of single runs. Because of this, the approach followed in this work can be a useful tool to examine whether the evolution is globally impeded or not by the observation by using common ideas in the context of an atom-field interaction.

In Sec. II we discuss the engineering of the decay of a metastable system and the evolution equations that govern it. In Sec. III we consider two different strategies to monitor the decay. We also find the way the observation affects the evolution equations. In Sec. IV we discuss the appearance of the Zeno effect, analyzing the similarities and differences with the purely coherent Zeno effect. In Sec. V we find the conditions under which the anti-Zeno effect can occur. Finally in Sec. VI we compare the approach followed in this work (based on the Bloch equations) to a different strategy based on the quantum trajectories.

\section{ENGINEERED DECAY}

In Fig. 1 we illustrate the three-level scheme in the $\mathrm{V}$ configuration that will lead to the engineered decay of a given metastable level $|1\rangle$. The state $|1\rangle$ is coupled to level $|0\rangle$ by a resonant laser field. On the other hand, the state $|0\rangle$ is also coupled by another resonant laser field to level $|2\rangle$. This level $|2\rangle$ is unstable and decays to $|0\rangle$ at rate $A_{2}$. This arrangement actually coincides with one of the examples of tailored decay analyzed in Ref. [9]. Moreover, it is worth noting that this is also the arrangement used to demonstrate the coherent Zeno effect [5]. In what follows, for numerical evaluations we will use realistic parameter regimes agreeing with the experimental ones used in Ref. [5] as reported in Ref. [16].

The unobserved evolution of the three-level system is given by the master equation (in the interaction picture and units in which $\hbar=1$ )

$$
\dot{\rho}=-i[H, \rho]-\frac{A_{2}}{2}(|2\rangle\langle 2|\rho+\rho| 2\rangle\langle 2|-2| 0\rangle\langle 2|\rho| 2\rangle\langle 0|),
$$

where $\rho$ is the density matrix and

$$
H=\frac{\Omega_{1}}{2}(|1\rangle\langle 0|+| 0\rangle\langle 1|)+\frac{\Omega_{2}}{2}(|2\rangle\langle 0|+| 0\rangle\langle 2|),
$$

where $\Omega_{1}$ and $\Omega_{2}$ are the Rabi frequencies for the corresponding transitions. This three-level dynamics has been well studied in different parameter regimes $[11-15]$. Here we will focus on the case $A_{2}, \Omega_{2} \gg \Omega_{1}$. This condition introduces two time scales that allow us remove rapid transients invisible in the coarse-grained time scale of interest. In such a case the equations of motion can be simplified by considering that the faster variables (essentially the variables associated with the transition $|0\rangle \leftrightarrow|2\rangle$ ) are always in their steadystate values that adiabatically follow the slower evolution of the rest of variables. As can be seen in Ref. [11] this procedure leads to the following closed set of equations for the variables associated with the transition $|0\rangle \leftrightarrow|1\rangle$

$$
\begin{gathered}
\dot{u}=-\frac{\gamma}{2} u, \\
\dot{v}=-\frac{\gamma}{2} v-\Omega_{1}\left(1+\frac{A_{2}^{2}}{A_{2}^{2}+2 \Omega_{2}^{2}}\right) P+\Omega_{1} \frac{A_{2}^{2}}{A_{2}^{2}+2 \Omega_{2}^{2}}, \\
\dot{P}=\frac{\Omega_{1}}{2} v,
\end{gathered}
$$

where $P=\langle 1|\rho| 1\rangle$ is the population of level $|1\rangle$, the variables $u, v$ are defined by

$$
\langle 0|\rho| 1\rangle=\frac{1}{2}(u+i v),
$$

and the parameter $\gamma$ is

$$
\gamma=\frac{\Omega_{2}^{2}}{A_{2}} .
$$

If the atom is initially in the state $|1\rangle$ the solution to these equations is $u=0$ and

$$
\begin{gathered}
v=\frac{\Omega_{1}}{\lambda_{+}-\lambda_{-}}\left(e^{-\lambda_{+} t}-e^{-\lambda_{-} t}\right), \\
P=\frac{\Omega_{1}^{2} / 2}{\lambda_{+}-\lambda_{-}}\left(\frac{1}{\lambda_{-}} e^{-\lambda_{-} t}-\frac{1}{\lambda_{+}} e^{-\lambda_{+} t}\right)+1-\frac{\Omega_{1}^{2} / 2}{\lambda_{+} \lambda_{-}},
\end{gathered}
$$

where 


$$
\lambda_{ \pm}=\frac{\gamma}{4} \pm \frac{1}{2} \sqrt{\frac{\gamma^{2}}{4}-4 \Omega_{1}^{2} \frac{A_{2}^{2}+\Omega_{2}^{2}}{A_{2}^{2}+2 \Omega_{2}^{2}}} .
$$

This solution demonstrates the irreversible decay of the previously metastable level $|1\rangle$. For example, if $\Omega_{2} \gg A_{2}$ we have $\lim _{t \rightarrow \infty} P \simeq 0$ and the system eventually leaves completely the initial state $|1\rangle$.

As we have mentioned above, this is an example of the idea of reservoir engineering [10]. The major advantage of this strategy is that the decaying parameters can be easily controlled via the intensities of the driving fields. In particular, it is possible to control the duration of the quadratic period suitable for the Zeno effect. This period lasts as long as $\lambda_{ \pm} t \ll 1$ (provided that $\lambda_{+} \lambda_{-} \neq 0$ ) and its duration depends on the Rabi frequencies $\Omega_{1}, \Omega_{2}$. When $\lambda_{ \pm} t \ll 1$ the survival probability can be approximated by

$$
P \simeq 1-\frac{1}{4} \Omega_{1}^{2} t^{2} .
$$

As we might have anticipated, the evolution during this initial stage solely depends on the Rabi frequency associated with the coherent $|1\rangle \leftrightarrow|0\rangle$ oscillation.

For definiteness, throughout this work we will always consider the common situation $A_{2} \gg \Omega_{2}$. In such a case the equations of motion (2.3) can be further simplified in the form

$$
\begin{gathered}
\dot{u}=-\frac{\gamma}{2} u, \\
\dot{v}=-\frac{\gamma}{2} v-2 \Omega_{1} P+\Omega_{1}, \\
\dot{P}=\frac{\Omega_{1}}{2} v,
\end{gathered}
$$

whose solutions are of the form (2.6) with

$$
\lambda_{ \pm}=\frac{\gamma}{4} \pm \frac{1}{2} \sqrt{\frac{\gamma^{2}}{4}-4 \Omega_{1}^{2}} .
$$

Concerning the long-time behavior, we have $\lim _{t \rightarrow \infty} P \simeq 1 / 2$ and when the steady state is reached the atom can be found in the initial level with $50 \%$ probability. This would be the same result of decaying into an infinite-temperature reservoir.

Before continuing we recall the conditions under which this arrangement serves to demonstrate the coherent Zeno effect. It is known that the fluorescence photons emitted in the transition $|0\rangle \leftrightarrow|2\rangle$ serve to detect the occurrence of the $|1\rangle \rightarrow|0\rangle$ transition [5,14]. A meaningful measurement of the population of $|1\rangle$ is obtained provided that $\gamma \gg \Omega_{1}$ [13]. In such a case we have from Eq. (2.10) that

$$
\lambda_{+} \simeq \frac{\gamma}{2}, \quad \lambda_{-} \simeq 2 \frac{\Omega_{1}^{2}}{\gamma} .
$$

Since $\lambda_{+} \gg \lambda_{-}$the survival probability in Eq. (2.6) can be well approximated by

$$
P \simeq \frac{1}{2}\left(1+e^{-2 \Omega_{1}^{2} t / \gamma}\right) .
$$

In the limit of an arbitrarily accurate observation $\Omega_{1} / \gamma \rightarrow 0$ and $P \rightarrow 1$ for finite $\Omega_{1} t$. This is the coherent Zeno effect experimentally demonstrated in Ref. [5].

Let us stress that in our case the primary role of the transition $|0\rangle \leftrightarrow|2\rangle$ is to produce and control the irreversible decay of the level $|1\rangle$. Therefore we are interested in the case in which $\Omega_{1} / \gamma$ is finite and not too close to zero so that during the time scale of the experiment there is time enough for $|1\rangle$ to decay. In other words, the engineered decay we are interested in is actually caused by an unsharp observation of the population of $|1\rangle$. This is referred to as the partial Zeno effect [15].

\section{OBSERVATION OF THE DECAY}

All preceding comments refer to the unobserved evolution of the three-level system. In order to test the Zeno effect it is necessary to monitor the population of $|1\rangle$. We will examine two different measuring strategies: pulsed and continuous.

By pulsed we mean that an ideal, instantaneous measurement is repeated at some specific instants $t_{n}=n \delta t$, where $n$ $=0,1, \ldots$. Between measurements the system evolves freely according to the unobserved dynamics just analyzed and given by the master equation (2.1).

By continuous we mean that the measurement (i.e., the coupling to the apparatus) coexists with the system dynamics all the time. Therefore, the evolution is no longer given by Eq. (2.1) because of the continuous backaction on the system caused by the observation.

To some extent, the pulsed observation fits more with what is usually understood as a quantum measurement (sudden irruption on the evolution of the system). On the other hand, the continuous observation allows us to obtain analytical formulas supporting a throughout analysis of these phenomena.

\section{A. Pulsed observation}

We begin by considering the pulsed observation. For simplicity we assume that during the measurements the decay of $|1\rangle$ is switched off. As a matter of fact, this can be easily achieved simply by switching off the field driving the $|0\rangle \leftrightarrow|1\rangle$ transition $\left(\Omega_{1}=0\right)$. This is another advantage of engineered decay which is not allowed for natural decaying systems. We further assume that the measurements are fully efficient and accurate so that a complete effective reduction of the measured system takes place. This implies that the observed survival probability $\mathcal{P}$ is given by

$$
\mathcal{P}\left(t_{n}\right)=[P(\delta t)]^{n} .
$$

More specifically, this is the probability that all the $n$ measurements (not only the last one) give that the atomic state is $|1\rangle$. 
Concerning the practical implementations of this strategy we note that the very same three-level structure in Fig. 1 can accommodate it without involving additional apparatus. After a period $\delta t$ of unobserved evolution the measurement begins by switching off the laser field driving the $|0\rangle \leftrightarrow|1\rangle$ transition $\left(\Omega_{1}=0\right)$. The Rabi frequency $\Omega_{2}$ might be also varied if necessary. Then one waits for the appearance (or not) of fluorescence photons from the $|0\rangle \leftrightarrow|2\rangle$ transition. The wait must last enough so that it is clear that such photons have appeared (the system was certainly not in $|1\rangle$ ) or that they will never appear (the system is certainly in $|1\rangle$ ). The waiting time depends on $\Omega_{2}$ and $A_{2}$. After this measurement stage, the original values of $\Omega_{1}, \Omega_{2}$ are resumed. The unobserved dynamics is recovered during another time interval $\delta t$, until a new measurement begins, and so on.

We think it is worth noting that the transition $|0\rangle \leftrightarrow|2\rangle$ can serve alternatively for two different purposes: decay engineering and measurement.

\section{B. Continuous observation}

In this case the original evolution and the measurement are not separated in time and both coexist during the whole process. This means that the evolution equations for the system are necessarily altered since they must accommodate the effect of the measurement.

The continuous observation of the population of level $|1\rangle$ can be suitably taken into account by adding a new term to the master equation (2.1),

$$
\begin{aligned}
\dot{\rho}= & -i[H, \rho]-\frac{A_{2}}{2}(|2\rangle\langle 2|\rho+\rho| 2\rangle\langle 2|-2| 0\rangle\langle 2|\rho| 2\rangle\langle 0|) \\
& -\frac{\Gamma}{2}(|1\rangle\langle 1|\rho+\rho| 1\rangle\langle 1|-2| 1\rangle\langle 1|\rho| 1\rangle\langle 1|),
\end{aligned}
$$

where $\Gamma$ is a parameter representing the time resolution of the observation. As a matter of fact, it has been shown that pulsed and continuous observations lead to similar results when $\delta t=4 / \Gamma[17]$.

The continuous measurement can be easily implemented by coupling $|1\rangle$ to an auxiliary decaying level and detecting the possible emission of photons when the auxiliary level decays $[5,14]$. It has been shown in Ref. [13] that such state detection is suitably described by the additional term in Eq. (3.2).

This modification of the master equation leads to an additional term of the form $-\Gamma\langle j|\rho| k\rangle / 2$ in the equations of motion for the matrix elements $\langle j|\dot{\rho}| k\rangle$ with $j=1$ or $k=1$ (excluding the case $j=k=1$ ). The same adiabatic elimination of fast variables discussed above leads in this case to the following closed set of equations for the variables associated with the $|1\rangle \leftrightarrow|0\rangle$ transition:

$$
\begin{gathered}
\dot{u}=-\frac{1}{2}\left(\Gamma+\frac{\Omega_{2}^{2}}{A_{2}+\Gamma}\right) u, \\
\dot{v}=-\frac{1}{2}\left(\Gamma+\frac{\Omega_{2}^{2}}{A_{2}+\Gamma}\right) v-\Omega_{1}(1+F) \mathcal{P}+\Omega_{1} F,
\end{gathered}
$$

$$
\dot{\mathcal{P}}=\frac{\Omega_{1}}{2} v,
$$

where

$$
F=\frac{A_{2}^{2}+\Omega_{2}^{2}}{A_{2}^{2}+2 \Omega_{2}^{2}}-\frac{A_{2} \Omega_{2}^{2}}{\left(A_{2}+\Gamma\right)\left(A_{2}^{2}+2 \Omega_{2}^{2}\right)} .
$$

For the sake of simplicity we are denoting the coherences $u$, $v$ in this observed case with the same symbols used for the unobserved one. As is discussed in the Introduction, in this case $\mathcal{P}=\langle 1|\rho| 1\rangle$ is the probability that the atom is in $|1\rangle$ irrespective of the history of the measurement outcomes. Therefore, the meaning of this survival probability is slightly different from $\mathcal{P}\left(t_{n}\right)$ in Eq. (3.1).

These equations can be solved without difficulties. However, in order to gain insight we will consider the usual situation where $A_{2} \gg \Gamma, \Omega_{2}$. This allows us to simplify Eq. (3.3) on the form

$$
\begin{gathered}
\dot{u}=-\frac{1}{2}(\gamma+\Gamma) u, \\
\dot{v}=-\frac{1}{2}(\gamma+\Gamma) v-2 \Omega_{1} \mathcal{P}+\Omega_{1}, \\
\dot{\mathcal{P}}=\frac{\Omega_{1}}{2} v .
\end{gathered}
$$

The solution when the initial state is $|1\rangle$ is given by Eqs. (2.6) with

$$
\lambda_{ \pm}=\frac{1}{4}(\gamma+\Gamma) \pm \frac{1}{2} \sqrt{\frac{1}{4}(\gamma+\Gamma)^{2}-4 \Omega_{1}^{2}} .
$$

We can appreciate that under these conditions the equations of motion for the observed system coincide with the corresponding ones for the unobserved evolution (2.9) with the only difference of replacing $\gamma$ by $\gamma+\Gamma$. It is worth noting that the effects of the decaying mechanism (represented by $\gamma$ ) and the measuring apparatus (represented by $\Gamma$ ) are exactly the same: the randomization of the atomic-dipole phase. We can resort to the analysis carried out in Sec. II to explain why measurement and decay have the same dynamical description in this case: the decay is actually caused by a partial Zeno effect.

\section{COHERENT VERSUS INCOHERENT ZENO EFFECT}

Armed with the results of the preceding sections we can analyze the occurrence of the Zeno effect in engineered decay. As we have mentioned in the Introduction, we might forecast that in order to stop the decay of $|1\rangle$ it would be enough to halt the coherent transition from $|1\rangle$ to $|0\rangle$. This would be supported by the short-time evolution (2.8) which only depends on $\Omega_{1}$. Accordingly, we would expect the Zeno effect when $\Gamma \gg \Omega_{1}$ for the continuous measurement or $\Omega_{1} \delta t \ll 1$ for the pulsed observation, which are the condi- 


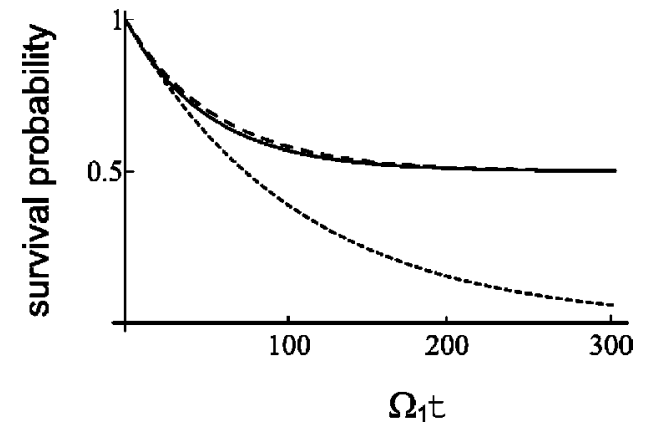

FIG. 2. Survival probability as a function of $\Omega_{1} t$ for $\Omega_{2}$ $=10^{4} \Omega_{1}, A_{2}=10^{6} \Omega_{1}, \Gamma=10 \Omega_{1}$, and $\delta t=4 / \Gamma$. We have represented the unobserved decay by a solid line, the observed decay via pulsed measurement by a dotted line, and the observed decay via continuous measurement by a dashed line. It can be seen that there is no Zeno effect.

tions for a coherent Zeno effect.

However, this is not always the case. To show this more clearly let us consider the case $\gamma \gg \Gamma \gg \Omega_{1}$. In Fig. 2 we have represented the survival probability. It clearly shows the lack of the Zeno effect since the decay is not stopped at all. Equations (2.6) and (3.6) confirm that the evolution under continuous observation tightly follows the unobserved decay (2.12)

$$
\mathcal{P} \simeq P \simeq \frac{1}{2}\left(1+e^{-2 \Omega_{1}^{2} t / \gamma}\right) .
$$

We can see also in Fig. 2 that the result of the pulsed observation is slightly different from Eq. (4.1) since the pulsed observation gives, when $\delta t \rightarrow 0$,

$$
\mathcal{P}\left(t_{n}\right) \simeq e^{-\Omega_{1}^{2} t_{n} / \gamma}
$$

This discrepancy may be ascribed to the facts that $P \rightarrow 1 / 2$ instead of $P \rightarrow 0$ and also that $\mathcal{P}\left(t_{n}\right)$ represents the probability that the measurement finds always the atom in the state $|1\rangle$.

According to the preceding reasonings, the lack of the Zeno effect might be regarded as paradoxical since the measurement is frequent enough to completely stop the Rabi oscillation $|1\rangle \leftrightarrow|0\rangle$. Nevertheless, this behavior can be easily explained in purely dynamical terms if we look directly at the evolution equations (3.5). If $\gamma \gg \Gamma$ the consequences of the measurement on the dynamics of the system are negligible, irrespective of the relative relation between $\Omega_{1}$ and $\Gamma$. In other words, the phase randomization caused by $\gamma$ overrides the effect of $\Gamma$.

In order to obtain the Zeno effect the arrangement must satisfy the necessary conditions $\Gamma,(\delta t)^{-1} \gg \gamma, \Omega_{1}$. For example, we can examine the regime $\Gamma \gg \gamma \gg \Omega_{1}$. In such a case we have from Eq. (3.6)

$$
\lambda_{+} \simeq \frac{\Gamma}{2}, \quad \lambda_{-} \simeq 2 \frac{\Omega_{1}^{2}}{\Gamma},
$$

so that $\lambda_{+} \gg \lambda_{-}$and the observed survival probability for the continuous observation can be well approximated by

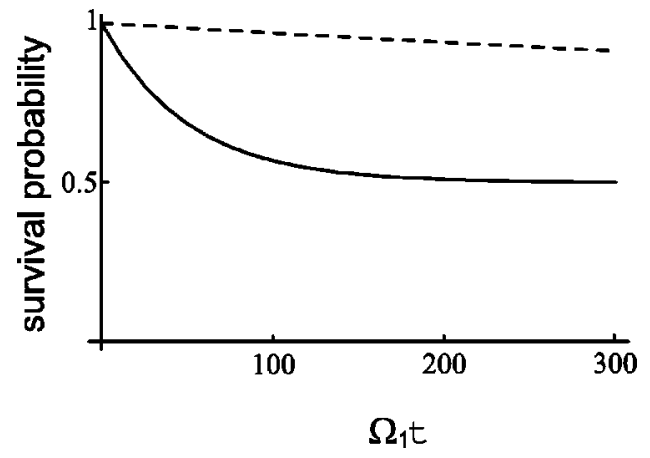

FIG. 3. Same as Fig. 2 for $\Omega_{2}=10^{4} \Omega_{1}, A_{2}=10^{6} \Omega_{1}, \Gamma=3$ $\times 10^{3} \Omega_{1}$, and $\delta t=4 / \Gamma$. The Zeno effect is clearly noticeable. Continuous and pulsed measurement coincide.

$$
\mathcal{P} \simeq \frac{1}{2}\left(1+e^{-2 \Omega_{1}^{2} t / \Gamma}\right)
$$

Since $\Gamma \gg \gamma$ the observed population decays slower than the unobserved one. In Fig. 3 we have represented both the observed and unobserved populations as a function of time showing the Zeno effect.

As we can see in the evolution equations (3.5), when $\Gamma$ $\gg \gamma$ the randomization of the dipole phase is due solely to the measurement process. If this randomization is fast enough, i.e., $\Gamma \gg \Omega_{1}$, the observation prevents the transition $|1\rangle \rightarrow|0\rangle$, the spontaneous decay is halted, and the atom remains at level $|1\rangle$.

\section{ANTI-ZENO EFFECT IN ENGINEERED DECAY}

In this section we show that this very same arrangement allows us to observe the so-called anti-Zeno effect. By the anti-Zeno effect we mean that the observed decay occurs faster than the unobserved one, i.e., the opposite of the Zeno effect [2].

This can occur, for example, when $\Omega_{1}>\gamma / 4$. In such a case from Eqs. (2.6), (2.9), and (2.10) the unobserved survival probability is the damped oscillation

$$
P \simeq \frac{1}{2}\left(1+\frac{\Omega_{1}}{\bar{\Omega}_{1}} \sin \left(\bar{\Omega}_{1} t+\delta\right) e^{-\gamma t / 4}\right),
$$

where $\bar{\Omega}_{1}=\sqrt{\Omega_{1}^{2}-\gamma^{2} / 16}$ and $\delta=\arg \left(\gamma / 4+i \bar{\Omega}_{1}\right)$. On the other hand, the observed decay when $\Gamma \gg \Omega_{1}$, for example, is given again by Eq. (4.4). If we compare Eqs. (4.4) and (5.1) we notice that the observation removes the oscillation and replaces the decaying constant $\gamma / 4$ by $2 \Omega_{1}^{2} / \Gamma$. In these conditions we will have the Zeno effect when $\Gamma$ $>8 A_{2}\left(\Omega_{1} / \Omega_{2}\right)^{2}$ as can be checked in Fig. 4.

On the other hand, the anti-Zeno effect occurs when $\Gamma$ $<8 A_{2}\left(\Omega_{1} / \Omega_{2}\right)^{2}$ as can be seen in Fig. 5: the observed population decays faster than the unobserved one. Similar results are obtained for pulsed observation replacing $\Gamma$ by $4 / \delta t$. This possibility of having both the Zeno and anti-Zeno effects on the same system, depending on the accuracy of the observation, agrees with the results of Ref. [3]. 


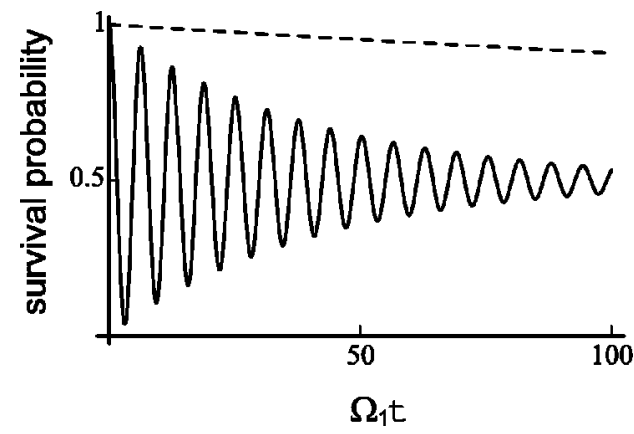

FIG. 4. Same as Fig. 2 for $\Omega_{2}=10 \Omega_{1}, A_{2}=10^{3} \Omega_{1}, \Gamma$ $=10^{3} \Omega_{1}$, and $\delta t=4 / \Gamma$. The Zeno effect is clearly noticeable. Continuous and pulsed measurement coincide.

\section{DISCUSSION AND CONCLUSIONS}

As we have pointed out in the Introduction, the Bloch equations used in this work represent the evolution of ensembles of independent systems that are observed simultaneously (or the average of repeated observations of a single system). On the other hand, an approach based on the evolution of individual systems may be regarded as being closer to the original idea of the Zeno effect. In this section we show explicitly that the observation of the evolution of a single atom leads us to the same conclusions obtained by means of the Bloch equations.

As we have discussed above, the engineered decay can be regarded as a partial Zeno effect. In the conditions leading to Eq. (3.5) we have that the effective atomic system is a twolevel atom (levels $|0\rangle$ and $|1\rangle$ ) that experiences a double measurement detecting the populations of $|0\rangle$ and $|1\rangle$. For the sake of simplicity, and to benefit from previous works, we can imagine that both are ideal pulsed measurements, which are repeated at rates $\gamma$ and $\Gamma$. For a two-level atom to measure the population of $|1\rangle$ is fully equivalent to measure the population of $|0\rangle$. Therefore we can regard the process simply as a measurement of the population of $|0\rangle$ repeated at rate $\gamma+\Gamma$.

The evolution of a single atom is a coherent oscillation interrupted by sudden jumps projecting the state onto $|0\rangle$ or $|1\rangle$, depending on the random outcome of the measurement. The outcome will form a stochastic sequence of the two possible results, $|0\rangle$ and $|1\rangle$. It has been shown that if $\gamma+\Gamma$ $\gg \Omega_{1}$ the outcomes are of the form of periods containing only one of the results [20]. The mean duration $T$ of these subsequences of identical results is $[20,23]$

$$
T=\frac{(\gamma+\Gamma)}{\Omega_{1}^{2}} .
$$

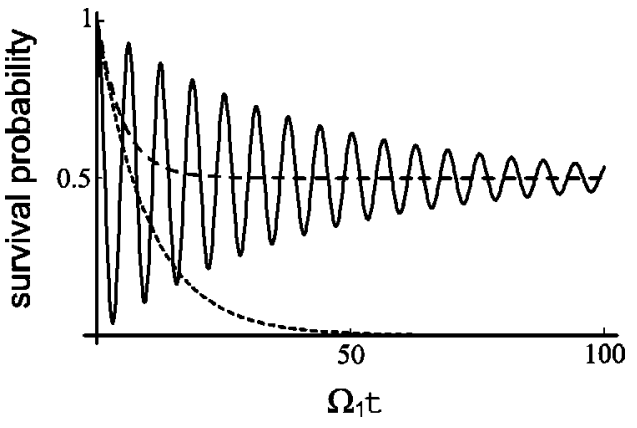

FIG. 5. Same as Fig. 2 for $\Omega_{2}=10 \Omega_{1}, A_{2}=10^{3} \Omega_{1}, \Gamma=10 \Omega_{1}$, and $\delta t=4 / \Gamma$. It can be seen that the observed population (dotted and dashed lines) decays faster than the unobserved one (solid line).

The Zeno effect would occur provided that $\Gamma \gg \gamma$. For increasing $\Gamma$ the initial period in which the initial state $|1\rangle$ endures becomes accordingly long in such a way that in the ideal limit $\Gamma \rightarrow \infty$ the system would remain always in the initial state $|1\rangle$.

Summarizing, in this work we focus on the occurrence of the incoherent Zeno effect in systems with engineered decay. To this end we have solved the equations of motion for the density matrix of a system experiencing simultaneously the mechanism that renders it unstable and the effect of the continuous observation. The decay is actually inhibited by halting a coherent Rabi oscillation. However, the decaying mechanism adds additional constraints.

The analysis simplifies if we take into account that the engineered decay is actually an incomplete or partial Zeno effect. Thus, the final evolution of the system results from the competition of two potential Zeno effects. As a consequence of this equivalence we have shown that the engineered decay and the observation have exactly the same effect on the system: i.e., the randomization of the atomic-dipole phase. As a matter of fact any of them can be regarded as being the measurement or as being the decaying mechanism. The explicit solutions of the complete equations of motion have allowed us to find a parameter regime where the anti-Zeno effect occurs.

All these points have been examined in a three-level system in the $\mathrm{V}$ configuration. We think it is worth stressing that one and the same arrangement serves to demonstrate different and even largely opposite phenomena, such as coherent, incoherent, and anti-Zeno effects, by means of a very simple change of parameters.
[1] D. Home and M.A.B. Whitaker, Ann. Phys. (N.Y.) 258, 237 (1997).

[2] A.M. Lane, Phys. Lett. 99A, 359 (1983); A. Luis and L.L. Sánchez-Soto, Phys. Rev. A 57, 781 (1998); S. Pascazio and P. Facchi, Acta Phys. Slov. 49, 557 (1999); A.G. Kofman and G.
Kurizki, ibid. 49, 541 (1999); Nature (London) 405, 546 (2000); M. Lewenstein and K. Rzążewski, Phys. Rev. A 61, 022105 (2000); O.V. Prezhdo, Phys. Rev. Lett. 85, 4413 (2000).

[3] P. Facchi, H. Nakazato, and S. Pascazio, e-print 
quant-ph/0006094.

[4] Y. Aharonov and M. Vardi, Phys. Rev. D 21, 2235 (1980); T.P. Altenmüller and A. Schenzle, Phys. Rev. A 48, 70 (1993); M. Kitano, ibid. 56, 1138 (1997); M. Kitano, K. Yamane, and T. Ikushima, ibid. 59, 3710 (1999); A.P. Balachandran and S.M. Roy, Phys. Rev. Lett. 84, 4019 (2000); L. Diósi, e-print quant-ph/010429.

[5] W.M. Itano, D.J. Heinzen, J.J. Bollinger, and D.J. Wineland, Phys. Rev. A 41, 2295 (1990).

[6] S. Slijkhuis, G. Nienhuis, and R. Morgenstern, Phys. Rev. A 33, 3977 (1986); K. Mølhave and M. Drewsen, Phys. Lett. A 268, 45 (2000); T. Nakanishi, K. Yamane, and M. Kitano, e-print quant-ph/0103034.

[7] B. Nagels, L.J.F. Hermans, and P.L. Chapovsky, Phys. Rev. Lett. 79, 3097 (1997).

[8] S.R. Wilkinson, C.F. Bharucha, M.C. Fischer, K.W. Madison, P.R. Morrow, Q. Niu, B. Sundaram, and M.G. Raizen, Nature (London) 387, 575 (1997).

[9] M.B. Plenio, P.L. Knight, and R.C. Thompson, Opt. Commun. 123, 278 (1996).

[10] J.F. Poyatos, J.I. Cirac, and P. Zoller, Phys. Rev. Lett. 77, 4728 (1996); C.J. Myatt, B.E. King, Q.A. Turchette, C.A. Sackett, D. Kielpinski, W.M. Itano, C. Monroe, and D.J. Wineland, Nature (London) 403, 269 (2000); Q.A. Turchette, C.J. Myatt, B.E. King, C.A. Sackett, D. Kielpinski, W.M. Itano, C. Monroe, and D.J. Wineland, Phys. Rev. A 62, 053807 (2000).
[11] H.J. Kimble, R.J. Cook, and A.L. Wells, Phys. Rev. A 34, 3190 (1986).

[12] V. Frerichs and A. Schenzle, Phys. Rev. A 44, 1962 (1991); E. Block and P.R. Berman, ibid. 44, 1466 (1991).

[13] M.J. Gagen and G.J. Milburn, Phys. Rev. A 47, 1467 (1993).

[14] W. Nagourney, J. Sandberg, and H. Dehmelt, Phys. Rev. Lett. 56, 2797 (1986); Th. Sauter, W. Neuhauser, R. Blatt, and P.E. Toschek, ibid. 57, 1696 (1986); J.C. Bergquist, R.G. Hulet, W.M. Itano, and D.J. Wineland, ibid. 57, 1699 (1986).

[15] A. Peres and A. Ron, Phys. Rev. A 42, 5720 (1990); T.F. Jordan, E.C.G. Sudarshan, and P. Valanju, ibid. 44, 3340 (1991).

[16] A. Beige and G.C. Hegerfeldt, Phys. Rev. A 53, 53 (1996).

[17] L.S. Schulman, Phys. Rev. A 57, 1509 (1998).

[18] T.P. Altenmüller and A.S. Schenzle, Phys. Rev. A 49, 2016 (1994).

[19] T.P. Spiller, Phys. Lett. A 192, 163 (1994); O. Alter and Y. Yamamoto, Phys. Rev. A 55, R2499 (1997); P.E. Toschek and C. Wunderlich, e-print quant-ph/0009021.

[20] A. Beige and G.C. Hegerfeldt, J. Phys. A 30, 1323 (1997).

[21] Chr. Balzer, R. Huesmann, W. Neuhauser, and P.E. Toschek, Opt. Commun. 180, 115 (2000).

[22] W.L. Power and P.L. Knight, Phys. Rev. A 53, 1052 (1996).

[23] C. Cohen-Tannoudji and J. Dalibard, Europhys. Lett. 1, 441 (1986); P. Zoller, M. Marte, and D.F. Walls, Phys. Rev. A 35, 198 (1987).

[24] A. Luis and J. Perina, Phys. Rev. Lett. 76, 4340 (1996). 